\title{
ESTUDO DO RESÍDUO GERADO NO PROCESSO DE DESLAMAGEM DO MINÉRIO DE FERRO
}

\author{
A. L. S. MARTINS ${ }^{1}$, L. YOKOYAMA ${ }^{1}$, F.V. FONSECA ${ }^{1}$, R.C. ALBUQUERQUE ${ }^{1}$ \\ ${ }^{1}$ Universidade Federal do Rio de Janeiro, Departamento de Processos Inorgânicos \\ E-mail para contato: adralmartins@ hotmail.com
}

\begin{abstract}
RESUMO - O reaproveitamento de resíduos sólidos industriais tem despertado grande interesse, já que tanto a legislação vigente quanto a relacionadas à disposição final destes resíduos tornam-se cada vez mais rigorosas e atuantes. Este estudo tem como objetivo caracterizar o resíduo de minério de ferro proveniente da etapa de deslamagem para sua posterior utilização como catalisador, sendo este a fonte de ferro na reação de Fenton Heterogêneo (POA) para a degradação de compostos orgânicos persistentes. A análise granulométrica foi realizada e concluiu-se que as frações com alto teor de hematita $\left(\mathrm{Fe}_{2} \mathrm{O}_{3}\right)$ e baixo teor de sílica foram das peneiras menor de 200 \#. Nessa faixa granulométrica o teor de hematita variou de 58,9 a $67,4 \%$, a área BET variou de 0,1345 a $1,3137 \mathrm{~m}^{2} / \mathrm{g}$. Neste estudo foi investigado a cinética de reação do resíduo com o mercaptobenzotiazol (MBT) e as isotermas de adsorção de Langmuir e Freundlich em $\mathrm{pH}$ 3, 5, 6 e 7. O resíduo pode ser utilizado nas reações de Fenton-Heterogêneo como catalisador nas faixas de $\mathrm{pH} 3$ e 5, pois nestes ocorrem as reações de oxidação e nos pHs 6 e 7 ocorrem a adsorção.
\end{abstract}

\section{INTRODUÇÃO}

A mineração é um dos setores básicos da economia do país, contribuindo de forma decisiva para o bem estar e a melhoria da qualidade de vida das presentes e futuras gerações, a partir do momento em que são responsáveis pela produção primária de vários materiais. A variedade de produtos químicos utilizados nas diversas etapas do beneficiamento de minérios cresceu com a necessidade de aumentar a eficiência dos processos e, dentre estes, ressaltam-se os produtos orgânicos, que são utilizados em elevadas concentrações. A remoção destes produtos permite aumentar o percentual de água de recirculação e diminuir a contaminação nas barragens de rejeito.

O reaproveitamento de resíduos sólidos industriais tem despertado grande interesse, com isso as empresas siderúrgicas vêem realizando diversas pesquisas em conjunto com universidades e instituições tecnológicas para o desenvolvimento de alternativas técnicas, ambiental e economicamente viáveis para a utilização dos resíduos gerados no seu processo produtivo.

As tecnologias convencionais de tratamento de efluente apresentam resultados poucos efetivos na degradação de poluentes persistentes e recalcitrantes (não biodegradáveis) e, para 
muitos destes tipos de substâncias, não são capazes de remover, seletivamente, quantidades tão pequenas destes contaminantes com baixos tempos reacionais e com alta eficiência de remoção. Diante disto, o uso dos Processos Oxidativos Avançados (POA) tem se tornado cada vez mais promissores e eficientes para promover degradação destas substâncias recalcitrantes e xenobióticas (ECKENFELDER, 2000).

Os POAs são processos que geram radicais hidroxila $(\bullet \mathrm{OH})$, altamente reativos, pois apresentam potencial padrão de oxidação elevado e igual a 2,8 V. Devido à sua alta reatividade, muitas vezes são capazes de oxidar várias substâncias orgânicas recalcitrantes, podendo levar a mineralização total ou parcial dos poluentes em água. Os POAs combinados a outras formas de tratamentos podem ser eficientes e economicamente viáveis (ELSELLAMI et al, 2009).

Neste estudo será investigada a utilização do rejeito do processo de deslamagem da indústria da mineração de ferro como catalisador de uma reação química de degradação de um composto orgânico persistente, esta substância orgânica é largamente utilizada nos processos de flotação de óxidos, na mineração do ouro e também como acelerador do processo de vulcanização das borrachas, o mercaptobenzotiazol (MBT), que também é um produto de degradação de um fungicida utilizado nas indústrias de couro e madeira (REEMTSMA et al, 1995), tem efeitos tóxicos sobre muitas bactérias, e sua presença inibe a biodegradação de outros compostos, incluindo alguns de seus produtos de degradação (VALDÉS et al, 2003). A mineralização parcial do MBT podem formar intermediários como: o MBO, MBI e outros (LIU et al, 2012).

O emprego de resíduos industriais no tratamento de efluentes líquidos seja como materiais adsorventes alternativos ou catalisadores é muito vantajoso, pois além de remover contaminantes de efluentes, minimiza o impacto ambiental e reduz os custos relativos à disposição do próprio resíduo em aterros.

\section{MATERIAIS E MÉTODOS}

A fonte de ferro utilizada na pesquisa é o resíduo em forma de pó da etapa de deslamagem do processo de beneficiamento do minério de ferro, um resíduo com altos teores de ferro, carbono e argila. O resíduo sólido utilizado neste trabalho foi doado pela VALE-Unidade de Araxá. O ponto de carga zero ( $\mathrm{pHzc}$ ) do resíduo 7. Os equipamentos utilizados para a caracterização do resíduo foram: Peneiras Vibratórias, Microscopia Eletrônica de Varredura (MEV), Raios X, Fluorescência de Raios X, DRX e espectrofotometria. O composto persistente estudado foi o MBT na concentração de $100 \mathrm{mg} / \mathrm{L}$, cuja estrutura molecular está na Figura 1.

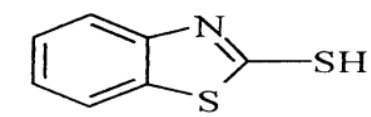

2-Mercaptobenzothiazole (MBT) 
Figura 1- Estrutura Molecular do MBT.

\subsection{Ensaios de Adsorção}

Os experimentos de adsorção foram conduzidos em triplicata, em frascos fechados colocados sob agitação em um shaker com controle de temperatura a $25^{\circ} \mathrm{C}+1$ e a $200+2 \mathrm{rpm}$.

Foram testadas as seguintes dosagens do resíduo: 0, 20, 40, 60, 80 e 100 g/L. Com o intuito de testar a influência do $\mathrm{pH}$ na adsorção realizou-se experimentos com diferentes valores de $\mathrm{pH}(3,5,6$ e 7) para cada uma das dosagens de resíduo e concentração do MBT de aproximadamente $100 \mathrm{mg} / \mathrm{L}$. $\mathrm{O} \mathrm{pH}$ da mistura foi mantido até o término de cada experimento com o ajuste adicionando $\mathrm{NaOH}(0,1 \mathrm{M})$ ou $\mathrm{H}_{2} \mathrm{SO}_{4}(0,1 \mathrm{M})$.

\subsection{Estudo da Cinética de Reação do MBT com o resíduo}

O estudo da cinética de adsorção foi realizado através do acompanhamento da concentração de MBT ao longo do tempo. Nas condições descritas anteriormente, foram retiradas alíquotas nos períodos de 3, 6, 21, 24, 30 e 41 horas para cada teste com o intuito de acompanhar o tempo de equilíbrio da solução.

A faixa de concentração da solução MBT utilizada foi assim escolhida devido à faixa de linearidade da Lei de Lambert-Beer. O tempo de equilíbrio foi determinado experimentalmente. As concentrações do MBT foram determinadas através de espectrofotometria $(\lambda=316 \mathrm{~nm})$.

\section{RESULTADOS E DISCUSSÕES}

\subsection{Caracterização do Resíduo - Raios X}

A Figura 2 apresenta o resultado da análise de raios $\mathrm{X}$ da amostra bruta do resíduo.

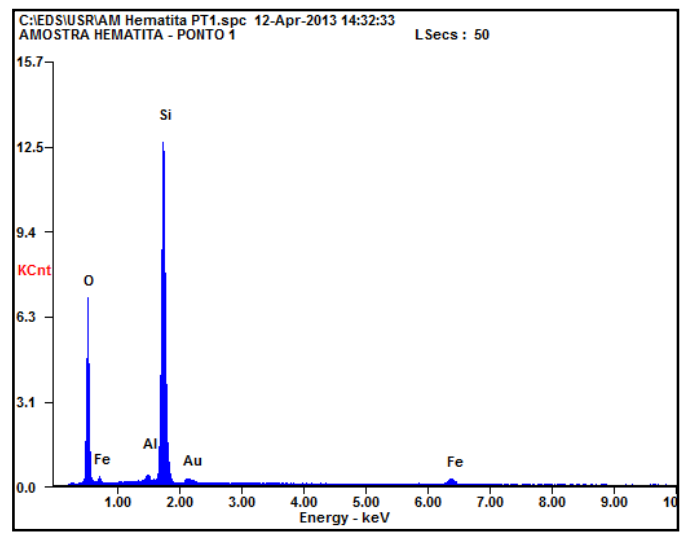

Figura 2- Difratograma de raios-X da hematita (resíduo). 
A análise do resíduo apresentou em sua composição a hematita $\left(\mathrm{Fe}_{2} \mathrm{O}_{3}\right)$, que será a fonte de ferro III para a reação de Fenton Heterogêneo, além de alto teor de sílica, como verificado na Figura 2. Rioja (2009) utilizou resíduo de siderurgia da etapa de desempoeiramento na Adsorção e peroxidação catalítica de surfactante aniônico, e constatou resultados semelhantes ao deste estudo.

\subsection{Análise Granulométrica da Amostra Bruta}

As análises de raios x da amostra bruta, verificou-se a necessidade da realização da etapa de peneiração do resíduo para uma melhor quantificação das frações mais significativas com relação ao teor de hematita e que seria mais efetiva na utilização do resíduo como catalisador. As frações de resíduo em cada peneira podem ser verificadas na Tabela 1, com as suas respectivas porcentagens.

Tabela 1 - Distribuição Granulométrica do resíduo bruto

\begin{tabular}{l|l|l|l|l}
\hline Amostras & $\begin{array}{l}\text { Mesh } \\
(\#)\end{array}$ & Abertura peneiras (mm) & Massa retida (g) & \% \\
\hline 1 & 60 & 250 & 2,325 & 0,5 \\
\hline 2 & 80 & 180 & 38 & 9,66 \\
\hline 3 & 100 & 150 & 9 & 2,28 \\
\hline 4 & 170 & 90 & 58 & 14,74 \\
\hline 5 & 200 & 75 & 58 & 14,74 \\
\hline 6 & 270 & 53 & 187 & 47,54 \\
\hline 7 & 325 & 45 & 34 & 8,64 \\
\hline 8 & Bandeja & $<45$ & 7 & 1,77 \\
\hline Total & & & $\mathbf{3 9 3 , 3 3}$ & \\
\hline
\end{tabular}

Tabela 2 - Caracterização do Resíduo - Fluorescência de Raios X

\begin{tabular}{l|l|l|l|l|l|l|l|l}
\hline Amostras & Mesh & $\begin{array}{l}\mathbf{M g O} \\
\mathbf{\%}\end{array}$ & $\begin{array}{l}\mathbf{A l}_{\mathbf{2}} \mathbf{O}_{\mathbf{3}} \\
\mathbf{\%}\end{array}$ & $\begin{array}{l}\mathbf{S i O}_{\mathbf{2}} \\
\mathbf{\%}\end{array}$ & $\begin{array}{l}\mathbf{P}_{\mathbf{2}} \mathbf{O}_{\mathbf{5}} \\
\mathbf{\%}\end{array}$ & $\begin{array}{l}\mathbf{C a O} \\
\mathbf{\%}\end{array}$ & $\begin{array}{l}\mathbf{M n O} \\
\mathbf{\%}\end{array}$ & $\begin{array}{l}\mathbf{F e}_{\mathbf{2}} \mathbf{O}_{\mathbf{3}} \\
\mathbf{\%}\end{array}$ \\
\hline Bruta & ---- & 0,04 & 0,79 & 50,6 & 0,04 & 0,07 & 0,20 & 47,1 \\
\hline 1 & 60 & $\mathrm{ND}$ & 0,27 & 56,5 & 0,03 & 0,15 & 0,08 & 41,3 \\
\hline 2 & 80 & 0,06 & 0,58 & 62,3 & 0,04 & 0,08 & 0,19 & 35,4 \\
\hline 3 & 100 & 0,07 & 1,3 & 55,5 & 0,06 & 0,09 & 0,34 & 41,1 \\
\hline 4 & 170 & $\mathrm{ND}$ & 0,30 & 66,3 & 0,02 & 0,06 & 0,18 & 58,9 \\
\hline 5 & 200 & 0,04 & 0,63 & $\mathbf{3 8 , 5}$ & 0,16 & 0,28 & 0,18 & 58,9 \\
\hline 6 & 270 & 0,05 & 0,55 & $\mathbf{3 0 , 9}$ & 0,04 & 0,06 & 0,18 & $\mathbf{6 7 , 0}$ \\
\hline 8 & 325 & 0,08 & 1,3 & $\mathbf{3 0 , 9}$ & 0,07 & 0,13 & 0,38 & $\mathbf{6 5 , 5}$ \\
\hline Fundo & fundo & 0,07 & 1,3 & $\mathbf{2 8 , 8}$ & 0,07 & 0,11 & 0,44 & $\mathbf{6 7 , 4}$ \\
\hline
\end{tabular}

De acordo com os resultados obtidos pela análise de fluorescência de Raio-X, tabela 2, observa-se que as frações do resíduo que foram mais significativas em teor de $\mathrm{Fe}_{2} \mathrm{O}_{3}$, foram a partir da peneira de 170\# (amostra 4) em diante. Perante o elevado teor de sílica apresentado na amostra $4\left(66,3 \%\right.$ de $\left.\mathrm{SiO}_{2}\right)$, trabalhou-se com a peneira de $200 \#$ em diante, faixas que apresentaram teores de sílicas entre 28,8 a 38,5\%. ARAÚJO (2009) utilizou hematita com um teor de ferro de $63 \%$ para a degradação de corante utilizando Fenton-Heterogêneo, embasando 
assim os resultados encontrados no resíduo em estudo, com teor de ferro variando de 58,8 a $67,4 \%$ e também justifica a faixa granulométrica escolhida.

Tabela 3 - Características texturais das frações de hematita

\begin{tabular}{|c|c|c|c|c|c|}
\hline Parâmetro & $\begin{array}{l}\text { Bruta } \\
\left(\mathrm{Fe}_{2} \mathrm{O}_{3}\right)\end{array}$ & $\begin{array}{l}200 \# \\
\left(\mathrm{Fe}_{2} \mathrm{O}_{3}\right)\end{array}$ & $\begin{array}{l}270 \# \\
\left(\mathrm{Fe}_{2} \mathrm{O}_{3}\right)\end{array}$ & $\begin{array}{l}325 \# \\
\left(\mathrm{Fe}_{2} \mathrm{O}_{3}\right)\end{array}$ & $\begin{array}{l}\text { fundo } \\
\left(\mathrm{Fe}_{2} \mathrm{O}_{3}\right)\end{array}$ \\
\hline Área BET (m $/ \mathrm{g})$ & 0,4117 & 0,1345 & 0,2397 & 1,0035 & 1,3137 \\
\hline Granulometria (mm) & ----- & 75 & 53 & 45 & $>45$ \\
\hline Volume de microporos $\left(\mathrm{cm}^{3} / \mathrm{g}\right.$ ) & ----- & $5,6 \times 10^{-4}$ & ----- & $8,0 \times 10^{-4}$ & $5,1 \times 10^{-4}$ \\
\hline Área de microporos (m $/ \mathrm{g})$ & ----- & 1,2005 & ----- & 1,3398 & 1,1874 \\
\hline $\begin{array}{l}\text { Diâmetro médio de } \\
\mathrm{A}^{\circ} \text { (Angstrom) }\end{array}$ & ----- & ------ & ----- & 34,7045 & ------ \\
\hline
\end{tabular}

Segundo Huang et al, (2001); Kwan e Voelke (2003); Matta (2007) a área superficial média encontrada para o mineral hematita é de $9-11 \mathrm{~m} / \mathrm{g}$, e as áreas superficiais encontradas nas frações analisadas de hematita (tabela 3) foram abaixo do valor apresentado na literatura. Neste estudo verificou-se que a área superficial do resíduo variou de 0,1345 a $1,3137 \mathrm{~m} / \mathrm{g}$, o que pode comprometer a capacidade adsortiva do mineral, o que também foi observado nos estudos de Rioja (2009) e Araújo (2009). Estes resultados foram perfeitamente aceitáveis uma vez que tal resíduo não é caracterizado como adsorvente e tão pouco recebeu algum tratamento para que tivesse propriedades adsortivas.

\subsection{Cinética de Reação do MBT com o resíduo}

A Figura 2 apresenta a cinética de reação do MBT com o resíduo em diferentes concentrações (0 a 100 g/L), agitação de 200 rpm, por 41 horas e com variação do pH.

(a)

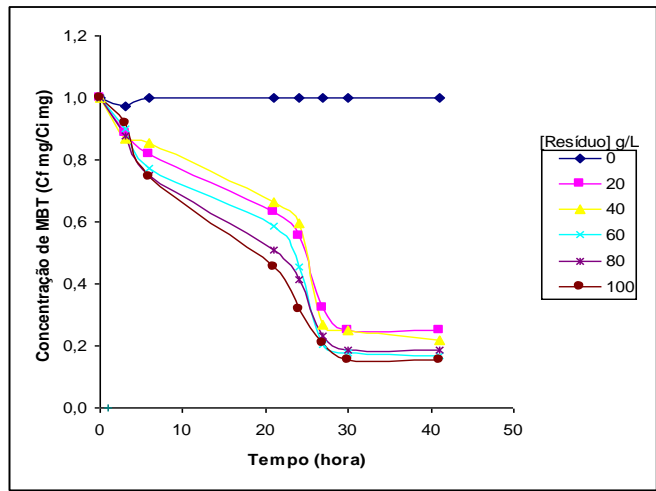

(b)

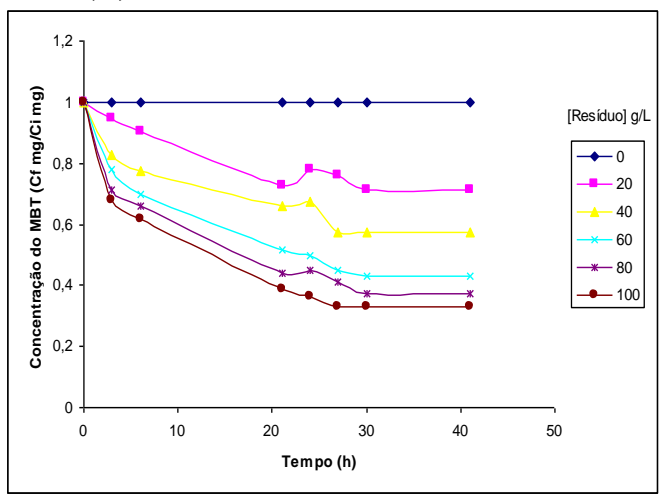


Figura 2. (a) Cinética da reação do MBT com resíduo em pH 3, (b) Cinética da reação do MBT com resíduo em pH 5, e sob variação da concentração do resíduo (g/L) e 200 rpm.

Ao analisar os resultados apresentados neste estudo, pode se observar que os melhores resultados de concentração do resíduo foram com $100 \mathrm{~g} / \mathrm{L}$ e em qualquer um dos pHs investigados.

\subsection{Estudo de Adsorção}

No estudo de adsorção foi investigado o comportamento do resíduo contendo hematita (adsorvente) em contato com a solução de MBT (100 mg/L) por um período de 41 horas em cada pH investigado. Avaliou se os modelos de adsorção de Langmuir e Freundlich ajustavam aos dados experimentais do sistema proposto (resíduo e solução de MBT) com variação de $\mathrm{pH}$, como apresentado nas Figuras 3 (a) e (b) e 4 (a) e (b). Para a construção das isotermas de Langmuir e Freundlich foram utilizadas as equações 1 e 2 , respectivamente.

$$
\begin{aligned}
& \frac{C_{e}}{q_{e}}=\frac{1}{K q_{m}}+\frac{1}{q_{m}} C_{e} \\
& q_{e}=K_{f} C_{e^{\frac{1}{n}}}
\end{aligned}
$$

(a)

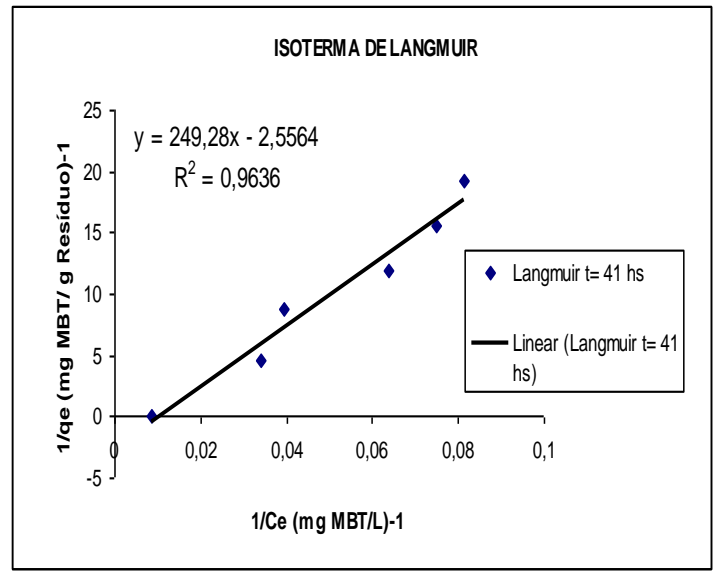

(b)

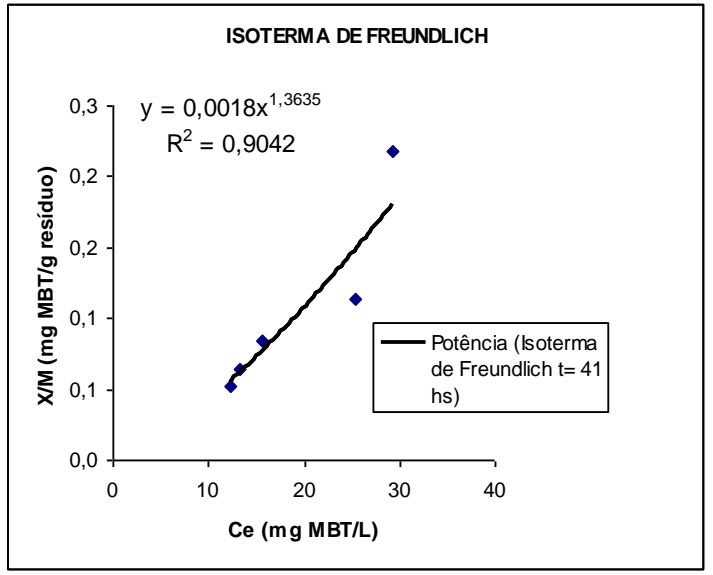

Figura 3- (a) Modelo de Langmuir e (b) Modelo de Freundlich, com o sistema em pH=3. 
(a)

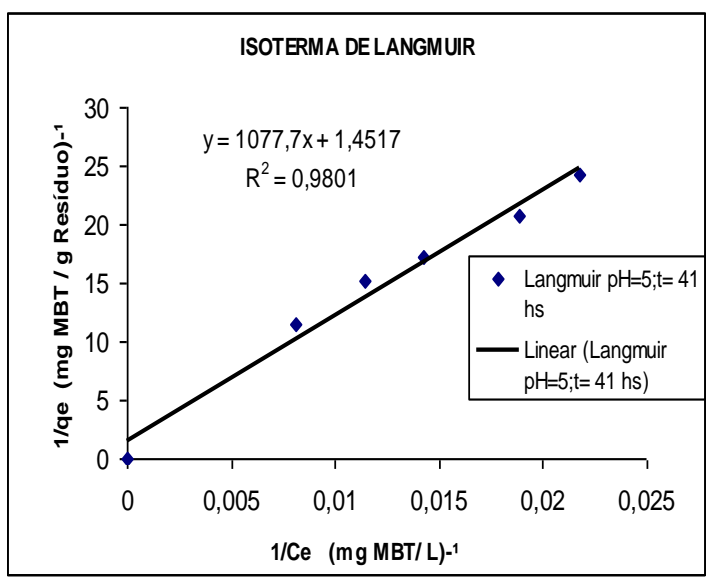

(b)

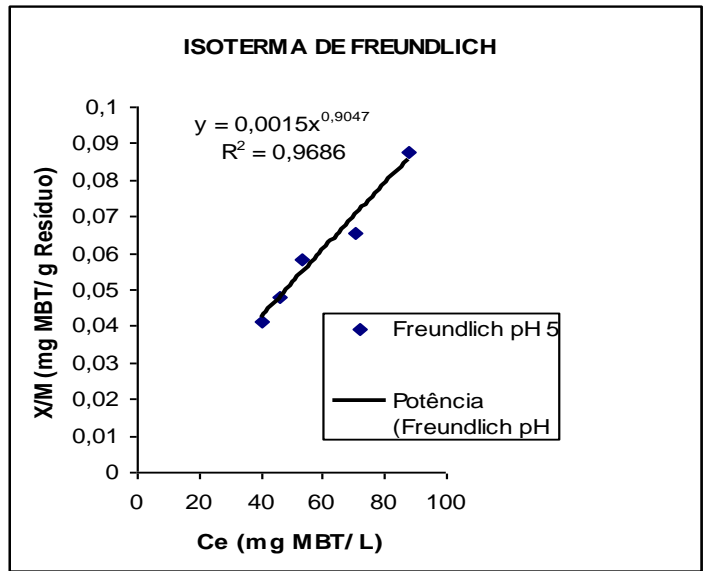

Figura 4- (a) Modelo de Langmuir e (b) Modelo de Freundlich. Sistema em pH 5.

A partir das interpolações gráficas realizadas nas Figuras de 3 e 4, obteve-se os coeficientes angulares e lineares em cada condição apresentada. Diante desses dados experimentais, coeficientes angulares e lineares, foram calculadas as constantes experimentais utilizando as equações 1 e 2 para cada modelo, nas condições em que cada sistema foi submetido, conforme apresentado nas tabelas 4 e 5.

Tabela 4: Valores das constantes experimentais das Isotermas de Langmuir

\begin{tabular}{l|l|l|l|l}
\hline $\mathbf{p H}$ & $\mathbf{R}^{2}$ & $\mathbf{q}_{\mathbf{m}}$ & $\mathbf{K}$ & $\mathbf{R}$ \\
\hline 3 & 0,963 & $-0,391$ & $-0,01$ & $\mathbf{1 , 1 4 0}$ \\
\hline 5 & 0,980 & 0,688 & $1,34 \times 10^{-3}$ & $\mathbf{0 , 9 5 2}$ \\
\hline
\end{tabular}

Tabela 5: Valores das constantes experimentais das Isotermas de Freundlich

\begin{tabular}{l|l|l|l}
\hline $\mathbf{p H}$ & $\mathbf{R}^{2}$ & $\mathbf{K f}$ & $\mathbf{n}$ \\
\hline 3 & 0,904 & $1,8 \times 10^{-3}$ & $\mathbf{0 , 7 3 3}$ \\
\hline 5 & 0,968 & $1,5 \times 10^{-3}$ & 1,105 \\
\hline
\end{tabular}

Em pH=3 verificou-se com os parâmetros calculados de Langmuir e Freundlich, observados nas Tabelas 4 e 5, não foram favoráveis aos modelos de adsorção e será investigado como ocorrem as reações de oxidação. Para $\mathrm{pH}=5$, a isoterma de Langmuir apresentou $\mathrm{R}=0,952$, indicando isoterma de adsorção favorável, mas neste caso será necessário também investigar a reação de oxidação por que a adsorção é mais favorável quanto mais próximo o valor de $\mathrm{R}$ estiver 
de zero, e neste estudo o valor está mais próximo de 1 . Nos pHs 6 e 7 foram observados a ocorrência de adsorção favorável.

\section{CONCLUSÃO}

Ensaios cinéticos mostraram que o tempo de equilíbrio do resíduo de mineração e a solução sintética de mercaptobenzotiazol foi de 41 horas. Assim, este tempo foi utilizado para a construção das isotermas de adsorção e também que a concentração ideal do resíduo foi de 100 g/l. A partir de ensaios experimentais, obteve-se o ponto de carga zero do resíduo é 7. Em pH=3, verificou-se com os parâmetros calculados de Langmuir e Freundlich, que não houve ajuste aos modelos de adsorção estudados e será investigado a reação de oxidação. Em pH=5 ocorreu adsorção e houve ajuste aos dois modelos mas também será necessário investigar a ocorrência de reações de oxidação. Nos pHs 3 e 5 foram realizados testes preliminares e observou a oxidação do MBT através da reação de Fenton-Heterogêneo. Nos pH 5, 6 e 7 ocorreu ajuste aos modelos de adsorção investigados.

\section{REFERÊNCIAS BIBLIOGRÁFICAS}

ARAÚJO, F. V. F., Estudo do processo Fenton Heterogêneo utilizando hematita $\left(\mathrm{Fe}_{2} \mathrm{O}_{3}\right)$ como catalisador na descoloração de soluções de corante reativo - Rio de Janeiro: Escola de Química/ UFRJ, 2008. Tese de doutorado.

ECKENFELDER, W. W., Industrial Water Pollution Control, $3^{\text {a }}$ ed., ed. McGraw Hill, 2000.

ElSEllami, L., CHARTRON, V., VOCANSON, F., CONCHON, P., FEliX, C., GUillard, C., RETAILLEAU, L. e HOUAS, A. Coupling process between solid-liquid extraction of amino acids by calixarenes and photocatalytic degradation. Journal of Hazardous Materials, v. 166, n. 2-3, p. 1195-1200, 2009.

LIU, G., ZENG, H., LU, Q., ZHONG, H., CHOI, P., XU, Z. Adsorption of mercaptobenzoheterocyclic compounds on sulfide mineral surfaces: A density functional theory study of structure-reactivity relations. Colloids and Surfaces A: Physicochem. 409, 1- 9, 2012.

HUANG, H-H.; LU, M-C.; CHEN, J-N. Catalytic decomposition of hidrogen peroxide and 2- chlorophenol with iron oxides. Water Research, v. 35, p. 2291-2299, 2001.

KWAN, W. P.; VOELKER, B. M. M. Rates of hydroxyl radical generation and organic compound oxidation im mineral-catalyzed Fenton-like systems. Environ. Sci. Technol. v. 37, 1150-1158, 2003.

MATTA, R.; HANNA, K.; CHIRON, S. Fenton-like oxidation of 2,4,6-trinitrotoluene using different iron minerals. Science of the Total Environment. V.385, 242-251, 2007.

REEMTSMA, T., FIEHN, O., KALNOWSKI, G., JEKEL, M. Microbial transformations and biological effects of fungicide-derived benzothiazotes determined in industrial waste water. Environ. Sci. Technol. 29 , 478-485, 1995.

RIOJA, J.D.C.C. Uso do Resíduo Siderúrgico na Adsorção e Peroxidação Catalítica de Surfactante Aniônico - Belo Horizonte: UFMG, 2009. Dissertação de Mestrado.

VALDES, H., SANCHES-POLO, M., ZAROR, C.A. Effect of ozonization on the activated carbon surface chemical properties an on 2-mercaptobenzthiazol adsorption. Lat. Am. Appl. Res. 33, 219-223, 2003. 